\title{
Lusioersily
}

\section{A Pilot Study of Gut-Brain Signaling after Octreotide Therapy for Unintentional Weight Loss after Esophagectomy}

Murphy, C. F., Stratford, N., Docherty, N. G., Moran, B., Elliott, J. A., Healy, M. L., Mcmorrow, J. P., Ravi, N.,

Goldstone, A. P., Reynolds, J. V., \& Le Roux, C. W. (2021). A Pilot Study of Gut-Brain Signaling after Octreotide Therapy for Unintentional Weight Loss after Esophagectomy. Journal of Clinical Endocrinology and Metabolism, 106(1), E204-E216. https://doi.org/10.1210/clinem/dgaa697

Link to publication record in Ulster University Research Portal

Published in:

Journal of Clinical Endocrinology and Metabolism

Publication Status:

Published (in print/issue): 31/01/2021

DOI:

10.1210/clinem/dgaa697

Document Version

Author Accepted version

\section{General rights}

Copyright for the publications made accessible via Ulster University's Research Portal is retained by the author(s) and / or other copyright owners and it is a condition of accessing these publications that users recognise and abide by the legal requirements associated with these rights.

\section{Take down policy}

The Research Portal is Ulster University's institutional repository that provides access to Ulster's research outputs. Every effort has been made to ensure that content in the Research Portal does not infringe any person's rights, or applicable UK laws. If you discover content in the Research Portal that you believe breaches copyright or violates any law, please contact pure-support@ulster.ac.uk. 


\section{A PILOT STUDY OF GUT-BRAIN SIGNALING AFTER OCTREOTIDE THERAPY FOR UNINTENTIONAL WEIGHT LOSS AFTER ESOPHAGECTOMY}

Conor F. Murphy, $\mathrm{MD}^{1,2}$; Nicholas Stratford, $\mathrm{MSc}^{1}$; Neil G. Docherty, $\mathrm{PhD}^{1}$; Brendan Moran, MD, $\mathrm{MCh}^{1}$; Jessie A. Elliott, MD, PhD, MRCS ${ }^{1,2}$; Marie-Louise Healy, MD, MRCPI ${ }^{3}$; Jason P McMorrow, $\mathrm{MD}, \mathrm{PhD}^{4}$; Narayanasamy Ravi, MD, FRCS ${ }^{2}$; Anthony P Goldstone, MD, PhD, MRCP ${ }^{5}$, John V. Reynolds, MD, FRCS ${ }^{2}$; Carel W. le Roux, MD, PhD, FRCPath ${ }^{1}$

1. Diabetes Complications Research Centre, Conway Institute of Biomolecular and Biomedical Research, University College Dublin, Dublin 4, Ireland.

2. National Oesophageal and Gastric Centre, Trinity Centre for Health Sciences, Trinity College Dublin and St. James's Hospital, Dublin 8, Ireland.

3. Department of Endocrinology, St. James's Hospital, Dublin 8, Ireland.

4. Department of Radiology, St. James's Hospital, Dublin 8, Ireland.

5. PsychoNeuroEndocrinology Research Group, Centre for Neuropsychopharmacology, Division of Psychiatry, and Computational, Cognitive, and Clinical Neuroimaging Laboratory, Department of Brain Sciences, Faculty of Medicine, Imperial College London, Hammersmith Hospital, London W12 0NN, United Kingdom.

(C) The Author(s) 2020. Published by Oxford University Press on behalf of the Endocrine Society. All rights reserved. For permissions, please e-mail: journals.permissions@oup.com jc.2020-01870 See https://academic.oup.com/endocrinesociety/pages/Author_Guidelines for Accepted Manuscript disclaimer and additional information. 
Correspondence: Professor Carel le Roux, Metabolic Medicine Group, Conway Institute of Biomolecular and Biomedical Research, University College Dublin, Dublin 4.

Email: carel.leroux@ucd.ie

Funding: Supported by a scholarship award from the Irish Cancer Society, Ireland, to CFM (CRS17MUR).

Disclosure summary: The authors have nothing to disclose. 


\begin{abstract}
Background

Recurrence-free patients after esophageal cancer surgery face long-term nutritional consequences, occurring in the context of an exaggerated post-prandial gut hormone response. Acute gut hormone suppression influences brain reward signaling and eating behavior. This study aimed to suppress gut hormone secretion and characterize reward responses and eating behavior among post-esophagectomy patients with unintentional weight loss.
\end{abstract}

\title{
Methods
}

This pilot study prospectively studied post-operative patients with $\geq 10 \%$ body weight loss (BWL) beyond one year who were candidates for clinical treatment with long-acting Octreotide (LAR). Before and after four weeks of treatment, gut hormone secretion, food cue reactivity (functional MRI), eating motivation (progressive ratio task), ad libitum food intake, body composition, and symptom burden were assessed.

\section{Results}

8 patients (7 male, age: mean \pm SD $62.8 \pm 9.4$ years, post-operative BWL: $15.5 \pm 5.8 \%$ ) participated. Octreotide LAR did not significantly suppress total post-prandial plasma GLP-1 response at four weeks $(P=0.08)$. Post-prandial symptom burden improved after treatment (Sigstad score median(range): $12(2-28)$ vs. $8(3-18), P=0.04)$, but weight remained stable (Pre:68.6 $\pm 12.8 \mathrm{~kg}$ vs. Post:69.2 $\pm 13.4 \mathrm{~kg}, P=0.13$ ). There was no significant change in brain reward system responses, during evaluation of high-energy or low-energy food pictures, nor their appeal rating. Moreover, treatment did not alter motivation to eat $(P=0.41)$ nor ad libitum food intake $(P=0.46)$. 


\section{Conclusion}

The protocol used made it feasible to characterize the gut-brain axis and eating behavior in this cohort. Inadequate suppression of gut hormone responses four weeks after Octreotide LAR administration may explain the lack of gut-brain pathway alterations. A higher dose or shorter interdose interval may be required to optimize the intervention.

Keywords: Esophagectomy; weight loss; gut-brain axis; enteroendocrine signaling; eating behavior; fMRI; food reward 


\section{INTRODUCTION}

Unintentional weight loss and gastrointestinal symptoms associated with malnutrition and impaired functional recovery are serious and common concerns for patients after esophagectomy, even among those who remain free from recurrent disease in the long-term (1-4). In addition to weight loss, malnourished patients may have sarcopenia and osteopenia (5-7). These issues accentuate the psychosocial burden experienced by patients post esophagectomy, impairing physical function and long-term health-related quality of life (HRQL) (8-10). Though there are myriad drivers of weight loss following esophageal cancer surgery, the impact of exaggerated post-prandial gut hormone signaling on appetite and satiety has emerged as a novel modifiable factor (11). This has more thoroughly been researched in bariatric surgery contexts and is relatively understudied postesophagectomy (12-14). However, targeted therapies, specifically modulating the gut-brain axis by suppressing gut hormone secretion, such as somatostatin analogues, have considerable theoretical rationale.

Scientific understanding of neural and hormonal pathways linking the gut to the brain is evolving. The dopaminergic mesolimbic pathway is the cornerstone of hedonic signaling, which together with hypothalamic homeostatic pathways control food intake and eating behavior, and this is influenced by feeding status, energy balance, perceived momentary value of food, and past learned experiences (1519). Taking a reductionist approach, eating behavior can be viewed as the sum of appetitive and consummatory behaviors, with the former representing, in this case, a positive motivation to seek food ('wanting'), and the latter, the actual consumption of food, which is intimately linked with, and driven by, anticipatory and actual food 'pleasure' or reward ('liking') $(20,21)$. Though methodologically challenging, it may be possible to measure appetitive behavior through techniques validated in bariatric research. Functional MRI and progressive ratio tasks (12), circumvent the limitations associated with self-reported methods, which are hypothesis-generating but prone to biases (22-24). Direct measurements of food intake and preferences represent a more robust and accurate approach to 
characterizing consummatory behavior $(25,26)$. Measuring both appetitive and consummatory components of eating behavior in parallel with gut hormone signaling may provide insight into changes in gut-brain signaling, and the impact of potential therapeutic strategies.

In the context of esophageal cancer surgery, the mechanisms by which gut hormones, such as glucagon-like peptide-1 (GLP-1) may influence eating behavior, symptomatology, and nutrition after surgery are becoming increasingly understood. Acute suppression of the exaggerated satiety gut hormone response using the somatostatin analogue Octreotide can increase ad libitum food intake by 50 per cent (27). Octreotide also increased motivation to eat in the acute setting, inducing a stronger desire to obtain a sweet-fat stimulus.(28) Several aspects of gut-brain signaling, however, remain unexplored in this operative context. First, the association between gut hormone suppression therapy post-esophagectomy and anticipatory food reward on fMRI is unknown. Second, how a short course of Octreotide therapy affects gut hormone signaling and eating behavior has not been reported. In various clinical contexts associated with weight loss and dumping syndrome, long-acting Octreotide (Octreotide LAR), which can reduce post-prandial symptoms and induce weight gain, has been used for decades $(29,30)$. However, there is a dearth of evidence regarding how best to employ this treatment paradigm to ameliorate excessive post-operative weight loss, currently limiting the viability of this targeted treatment approach. Parallel assessment of gut hormone signaling, food reward response, and eating behavior has not been performed previously but such a methodological approach may facilitate both a greater understanding of post-esophagectomy physiology and the therapeutic benefit of long-acting somatostatin analogue therapy.

It is within this context that this pilot study sought to: a) comprehensively characterize the gut-brain signaling pathway in a post-esophagectomy cohort, b) assess gut hormone and brain reward system food cue reactivity associated with a 4-week course of Octreotide LAR therapy, and c) determine the relationship between eating behavior and Octreotide LAR treatment. 


\section{MATERIALS AND METHODS}

\section{Participants}

We recruited patients who were disease-free at least 1 year post curative intent esophagectomy with gastric conduit reconstruction for esophageal cancer, who had $\geq 10 \%$ unintentional weight loss and were scheduled to receive Octreotide LAR as a trial of therapy to improve weight. All patients had a significantly raised Sigstad score (>7) or persistent lack of appetite. First treatment dose was given in the outpatient clinic, with a shared decision made with each patient based on whether to continue based on treatment response after four weeks. Response was assessed using self-perceived improvement based on questionnaire scores as well as objective changes in appetitive behaviour and weight. Exclusion criteria included: nutritional compromise due to overt issues such as anastomotic stricture with significant dysphagia or significant malabsorption of any cause, the presence of neuropsychiatric illness, the presence of alcohol or other substance misuse disorders, contraindications to the use of Octreotide LAR.

\section{Study Design and Protocol}

This study was approved by the regional research ethics committee (2017-07 (6)) and it was undertaken in accordance with the principles of ICH Good Clinical Practice with written, informed consent obtained from all participants. The study was prospectively registered on ClinicalTrials.gov (NCT03377660). Participants were studied on two occasions, before and four weeks after an intramuscular dose of 10mg Octreotide LAR (Figure 1), as used previously to treat dumping syndrome in the post-gastrectomy context [Penning, 2005 \#7], which was accompanied by a prophylactic course of pancreatic enzyme replacement therapy. At each visit, participants attended the Clinical Research Facility at 08:45 having completed a 12-hour fast, excluding water and regular medications. The protocol had four components, all of which were completed on the same day. These included (a) standardized mixed meal challenge, (b) functional MRI tasks, (c) progressive ratio task, and (d) ad libitum buffet meal. 


\section{Mixed meal challenge}

Venous blood samples were drawn before, and at 30,60,90,120, and 150 minutes after a $400 \mathrm{kcal}$ standard semi-solid mixed meal (160 g (184 mL): 61.0\% fat, $6.7 \%$ protein, 32.3\% carbohydrate). Using a $10 \mathrm{~cm}$ visual analogue scale recorded at each blood sampling time point, temporal patterns of hunger, nausea, desire to eat, prospective food consumption, fullness, and sleepiness were measured. A composite appetite score was also calculated based on VAS scores, based on the following formula: [hunger $+(100-$ fullness $)+$ desire to eat + prospective food consumption] $/ 4$ (31).

Each participant completed European Organization for Research and Treatment of Cancer (EORTC) HRQL questionnaires (General HRQL: QLQ-C30, and cancer-specific sections: QLQ-OES18 and QLQ-OG25) $(32,33)$, as well as a Sigstad post-prandial dumping syndrome questionnaire and a modified gastrointestinal symptom rating scale (32). The Sigstad score is a questionnaire utilized in the diagnosis and assessment of post-operative dumping syndrome, with a score greater than seven indicative of clinically significant dumping syndrome. Body weight and body composition were assessed using bio-electrical impedance analysis (BIA, Seca 515, Seca GMBH \& Co., Hamburg, Germany) with participants wearing light clothing, post voiding of urine, as per best practice $(34,35)$.

\section{Functional MRI Tasks}

Study participants then underwent both structural and functional magnetic resonance imaging (fMRI) scanning over a 60 minute period, from 180 - 240 minutes, following a previously described protocol $(12,36-38)$. During this period they performed a food picture evaluation fMRI task in which they rated the appeal of pictures of high-energy foods, low-energy foods and objects $(12,36-38)$, followed by a control auditory-motor-visual (AMV) task (38). For the food picture evaluation fMRI task, the effects of Octreotide LAR treatment on blood oxygen level dependent signal (BOLD) was examined by both a priori function region of interest (fROI) analysis (nucleus accumbens, caudate, putamen, 
amygdala, anterior orbitofrontal cortex (OFC), anterior insula) and whole brain analysis for the following picture contrasts: high-energy > low-energy food, high-energy food > object, low-energy food > object, nor any food (high-energy or low-energy food) $>$ object. A detailed description of the fMRI methodology is included in the Supplementary methods(39).

\section{Progressive Ratio Task}

Participants were asked at each visit to complete a computer-based progressive ratio task (PRT) following the fMRI, as per a previously described protocol [Goldstone, 2016 \#2957; Miras, 2012 \#3625]. This validated paradigm involves using a sweet-fat stimulus to gauge motivation or desire to eat. Each session lasted for up to 15 minutes (or upon cessation of the task by the participant themselves) and was performed once before and once after treatment with Octreotide LAR. Participants were asked to click the computer mouse in order obtain the candy reward reinforcer. The number of required clicks to receive the reward increased from 10, at the first stage, in a geometric increment of two $(10,20,40,80,160,320$ clicks etc.), with the final completed increment referred to as the 'PRT breakpoint'. At each stage, the computer program instructed participants to ingest a single reward. It then urged participants to continue clicking the mouse, if they so desired, to obtain the next reward. They could continue clicking or stop at any point when the arduousness of the task outweighed the perceived value of the food reward. Visual analogue scales were completed, and blood samples taken, immediately before the PRT and after the subsequent buffet meal.

\section{Ad libitum buffet meal}

The final test was a buffet meal paradigm, conducted in a research kitchen, which used a direct measures approach to assessing macronutrient intake and preference based upon a method created and validated by Geiselman et al (40). When recruited to the study, participants completed a screening food preference questionnaire, which was used to inform the 18 food items that comprised the personalized participant buffet meal for each visit. The selected foods represented six macronutrient 
groupings, of which three were high-fat and three were low-fat. Within each trio, the carbohydrate (complex and simple) and protein content varied. Quantities of each of the 18 foods were provided in excess to ensure there was no limit to ad libitum consumption, which could introduce bias.

Participants were not aware that macronutrient type and quantity ingestion was being measured, with foods weighted prior to testing. Instead, participants were informed that in order to optimize the postprandial stimulation of gut hormones, they should consume the foods they liked until they were comfortably satiated. This 45 minute study stage was followed by a final blood draw and VAS score completion. The foods remaining were then weighed covertly to ascertain the exact quantity consumed.

\section{Plasma analysis}

Once drawn, blood samples were placed on ice and carried to the laboratory in an adjacent room. They were immediately centrifuged at $2500 \mathrm{rpm}$ at $4{ }^{\circ} \mathrm{C}$ for 10 minutes, with plasma then stored at -80 ${ }^{\circ} \mathrm{C}$ to minimize sample degradation. Plasma total GLP-1 levels were measured by sandwich enzymelinked immunosorbent assay (ELISA) (GLP-1 Total ELISA, Merck Millipore, Darmstadt, Germany). The assay used was sensitive, validated to detect above 1.5 picomolar (pM) with a $50 \mu \mathrm{L}$ sample, and specific, having no cross-reactivity with GLP-2, GIP, glicentin, or oxyntomodulin. The intra- and inter-assay co-efficient of variation were $<2 \%$, and $<12 \%$, respectively. Plasma glucose and insulin were measured using an automated analyzer (ALINITY, Abbott Laboratories, IL, USA).

\section{Statistics}

\section{Power calculations}

This was a pilot study with the principal aim to determine feasibility of the protocol and effect size of the outcomes. When planning the study we used the PRT breakpoint as primary outcome as our previous data demonstrated that the difference in the response of matched pairs to Octreotide is normally distributed and is 88 with standard deviation of 47 (28). Were these values used, with a 
significance level of 0.05 and 0.9 power, then 5 participants would be needed to detect a difference with Octreotide LAR treatment.

\section{Statistical analysis}

GraphPad Prism (version 8.3) for macOS (GraphPad software (San Diego, CA, USA)), was used for data analysis. As per EORTC recommendations, a linear transformation of EORTC questionnaire responses into numeric values ranging from 0-100 was conducted, with rising scores indicating increased symptom severity or improved quality of life scores. Data were reported as mean \pm standard deviation, or median (range), as appropriate, unless otherwise specified. Area under the curve (AUC) was calculated using the trapezoidal rule. Inferential testing for within-group univariate comparisons involved paired Student's $t$ or Wilcoxon signed rank tests, as appropriate. To test for significant differences within individual variables over multiple timepoints, one-way repeated measures analysis of variance (ANOVA) with post-hoc Bonferroni's multiple comparisons test employed, unless otherwise stated. In the case of two or more variables followed over time, two-way repeated measures ANOVA with post-hoc Bonferroni multiple comparisons test used. Continuous variable interrelationships were determined using simple linear regression. Effect sizes are described as mean difference [95\% confidence intervals (CI)], and Cohen's d values. Statistical analyses were two-tailed, and $P$-values below an alpha level of 0.05 were deemed statistically significant. 


\section{RESULTS}

\section{Cohort characteristics}

The study cohort was demographically representative of a typical post-operative Western esophageal cancer population, with a mean age of 63 years, 7 (87.5\%) males, and 6 with adenocarcinoma $(75 \%)$ (Table 1). While pre-operatively participants tended to be overweight with a mean BMI of 27.2 $\mathrm{kg} / \mathrm{m}^{2}$, this fell to $22.4 \mathrm{~kg} / \mathrm{m}^{2}$ by the first study visit. At a median of 23.6 months post-operatively, the average weight loss from pre-operative weight was $15.5 \%$. Pathological and treatment characteristics are outlined in Table 1.

\section{Plasma glucagon-like peptide-1}

Although the shape of the post-prandial GLP-1 response curve was altered at the week 4 timepoint $(P=0.01)$, overall suppression with treatment as determined by the area under the curve was not significant $(P=0.08)$ (Figure 2). On post-hoc analysis, GLP-1 response at 30 minutes was lower after treatment $(P<0.001)$. This is corroborated by a trend towards the suppression of early AUC (up to 60 minutes post meal ingestion, $P=0.053$ ), but no evidence of a significant difference in overall AUC $(P=0.30)$. There was also a trend towards a reduction in peak post-prandial GLP-1 (median [IQR] week 0: 145 [93 - 192] pM vs. week 4: 135 [72 - 152] pM, $P=0.055)$. Post-mixed meal appetite and nausea VAS scores did not differ between visits (Supplementary Figure S4 (39)).

\section{Plasma glucose and insulin}

Post-prandial plasma glucose concentration curves were different at week 4 , relative to pre-treatment $(P=0.046)$, with a more sustained post-prandial glycemic response to 60 minutes post-prandially (week 0: $5.6(1.4) \mathrm{mmol} / \mathrm{L}$ vs. week 4: $7.5(2.1) \mathrm{mmol} / \mathrm{L}, P<0.001)$ (Figure $3 \mathrm{~A}$ ). Treatment was not associated with a reduction in fasting or peak post-prandial glucose concentrations (both $P>0.99$ ). There were no cases of biochemical reactive hypoglycemia before or after treatment, defined as a post-prandial glucose of $<2.8 \mathrm{mmol} / \mathrm{L}(41)$. 
Post-prandial plasma insulin concentrations were not significantly different at week 4, compared with week $0(P=0.69)$ (Figure 3B). However, there was a significant treatment $\mathrm{x}$ time effect, with a delayed peak insulin at week $4(P<0.001)$, with the concentration at 30 minutes, on post-hoc analysis, approximately two-fold higher pre-treatment (mean (SD), week 0: 97.2 (51.8) vs. week 4: 44.0 (33.7) $\mathrm{mU} / \mathrm{L}, P<0.001$ ), and the concentration at 60 minutes almost four-fold higher post-treatment (week 0 : 21.6 (11.2) vs. week 4: $79.5(38.3) \mathrm{mU} / \mathrm{L}, P<0.001)$.

\section{Body anthropometry and composition}

There were no significant changes in body weight after four weeks of treatment with Octreotide LAR (Table 2). There was an increase in waist-hip ratio (WHR) after four weeks of treatment (mean (SD), week 0: $0.88(0.1)$ vs. week 4: $0.90(0.1), P=0.02)$, although no change in absolute or relative body fat mass, or in skeletal muscle mass was observed.

\section{Eating behavior}

\section{Motivation to eat}

No difference was observed in appetitive behavior, as measured by PRT breakpoint (the last completed ratio), after four weeks of clinical treatment (median [IQR], week 0: 960 [400 - 1280] vs. week 4: 640 [320 - 1120], $P=0.41$ ) (Figure 4). Absolute number of clicks did not differ before and after treatment (mean (SD), week 0: 1479 (707) vs. week 4: 1251 (716) clicks, $P=0.52$ ) (Figure 4). These results occurred in the context of analogous pre-PRT plasma GLP-1 concentrations $(P=0.25)$ and VAS appetite ratings, the latter of which suggests no change in 'pleasure' to receive food associated with treatment $(P=0.46)$. More significant plasma GLP-1 suppression with Octreotide LAR correlated significantly with an increase in eating motivation as measured by total clicks during the PRT $\left(P=0.026, \mathrm{R}^{2}=0.59\right) \quad($ Supplementary Figure S5 (39)). 


\section{Ad libitum food intake and macronutrient preference}

There was no difference in total energy intake during the ad libitum buffet meal before and after treatment (mean (SD), week 0: 796 (280) kcal vs. week 4: 847 (229) kcal, $P=0.46$ ) (Figure 5A), nor any change in macronutrient selection $(P=0.26)$ (Figure 5B).

Importantly, $\%$ fat intake was not related to relevant symptoms, such as diarrhea $(P=0.70)$, loss of appetite $(P=0.58)$, and Sigstad score $(P=0.18)$, but was significantly associated with increased refluxlike symptoms $\left(P=0.009, \mathrm{R}^{2}=0.70\right)$.

\section{Functional MRI tasks}

In the food picture evaluation fMRI task, functional region of interest (fROI) analysis using 2-factor repeated-measures ANOVA, including ROI and treatment visit as within subject factors, there were no significant ROI $x$ visit interactions for the BOLD signal for any of the following contrasts: highenergy food vs. low-energy food $[\mathrm{F}(2.4,16.4)=0.713, P=0.525]$, high- or low-energy food vs. object $[\mathrm{F}(2.8,19.3)=1.62, P=0.314]$, high-energy food vs. object $[\mathrm{F}(2.8,19.5)=2.07, P=0.14]$, and lowenergy food vs. object $[\mathrm{F}(2.8,19.6)=0.411, P=0.73]$ (Figure 6A-D). There were also no significant main effects of visit for any of these picture contrasts, independent of ROI (see Figure 6 legend for statistics). Thus, treatment with Octreotide LAR had no significant effect of high-energy or lowenergy food cue reactivity.

For food picture appeal rating (relative to objects), there was also no significant energy density $\mathrm{x}$ visit interaction $[F(1,7)=2.396, P=0.166]$ nor main effect of visit (independent of energy density) [effect size mean \pm SEM $[95 \% \mathrm{CI}]-0.110 \pm 0.152[-0.470,0.252], \mathrm{F}(1,7)=0.515, P=0.496]$ (Figure 6F). 
Thus, treatment with Octreotide LAR had no effect on food appeal independent or dependent on energy density.

In whole-brain analysis for the food picture evaluation fMRI task, there were no clusters displaying significant differences in BOLD signal (voxel-wise false discovery rate (FDR) $\mathrm{P}<0.05$; nor clusterwise family wise error $(\mathrm{FWE}) \mathrm{Z}>2.1, \mathrm{P}<0.05)$ between visits for the high-energy $>$ low-energy food, high-energy food > object, low-energy food > object, nor any food (high-energy or low-energy food) $>$ object picture contrasts.

The control auditory-motor-visual (AMV) fMRI task was designed to account for non-specific BOLD signal changes between visits.(38) In fROI analysis, no significant differences in BOLD signal within any fROI was found comparing before and after treatment (ROI $x$ visit interaction: $\mathrm{P}=0.44$, main effect of visit: $\mathrm{P}=0.46$, Figure $6 \mathrm{E})$.

\section{Potential confounding variables}

There were no significant differences between visits in variables that could have confounded BOLD signal responses: pre-fMRI composite appetite VAS ratings (median (range), week 0: $6.6(2.4-7.7)$ vs. week 4:5.4 (2.7 -7.9), $P=0.64)$, pre-fMRI nausea and sleep VAS ratings (Supplementary Figure S4 (39)), BMI $(P=0.21), \%$ body fat mass $(P=0.58)$, pre-scan energy intake (400 kcal at mixed meal given to all participants), head motion during food picture evaluation fMRI task [average relative motion (mm per volume), median (IQR) week 0: $0.09(0.08,0.15)$, week 4: $0.14(0.08,0.16)$, Wilcoxon signed rank test $P=0.074$ ), with no participants having motion $>0.28 \mathrm{~mm}$ per volume]. 


\section{Post-prandial symptoms and health-related quality of life}

There were no significant differences in reported HRQL outcomes before vs. after treatment with Octreotide LAR, with global HRQL remaining stable $(P=0.94)$ (Table 3).

When comparing key appetite and nutrition-related patient-reported outcomes, Sigstad score was lower after treatment (median (range), week 0: 12 (2-28) vs. week 4: 8 (3-18), $P=0.039$ ) (Figure 7, Table 3). The degree of plasma GLP-1 suppression (delta AUC 0-150 mins) did not correlate with improvement in Sigstad score $\left(P=0.58, \mathrm{R}^{2}=0.05\right)$. However, EORTC symptom appetite loss severity scores did not change significantly with Octreotide LAR treatment: median (range) week 0: $66.7(0-$ 66.7), week 4: $0(0-66.7)(P=0.063)$ (Table 3$)$. 


\section{DISCUSSION}

This pilot study shows that it is feasible to characterize the gut-brain axis and its relation to food reward and eating behavior in a cohort post esophagectomy. Notwithstanding, 10mg Octreotide LAR was not sufficient to change gut hormone response, body weight, appetitive behavior or ad libitum food intake.

Somatostatin analogues have been used in surgical contexts to reduce post-prandial symptoms and can have beneficial nutritional effects, including the restoration of post-operative weight loss $(30,42)$. There has, however, historically been no method to systematically analyze the factors underlying their potential beneficial effects in the context of major upper gastrointestinal resection. This limits the ability to appropriately target treatment to patients who are most likely to derive benefit. Recent developments in our understanding of how alterations in gut-brain axis signaling may be implicated in the pathophysiology of post-esophagectomy symptomatology and malnutrition (11), should enable a more systematic approach to therapeutic interventions. Moreover, while studies at least suggest a compelling rationale for long-term nutritional benefit with the use of Octreotide LAR, through mitigation of the exaggerated post-operative gut hormone response, this remains to be proven.

In this study, the first to assess Octreotide LAR in this context, the scientific thesis was that Octreotide administration, shown previously to acutely inhibit GLP-1 secretion and increase ad libitum food intake among patients post esophagectomy, would suppress gut hormones and potentially facilitate weight regain. In this model, GLP-1 was the preferred marker to represent general postprandial gut hormone secretion. We anticipated that significant plasma GLP-1 suppression at the mixed meal challenge would also be associated with increased brain reward system food cue reactivity and eating behavioral changes. However, four weeks after a treatment dose of long-acting Octreotide, GLP-1 secretion, though blunted in the early post-prandial phase, was not sufficiently suppressed with the dosage used. Moreover, in addition to the absence of a change in brain reward 
system food cue reactivity and eating behavior, there were no significant changes in body weight or muscle mass. Given the suboptimal plasma GLP-1 suppression, the observations described herein cannot be asserted to reflect observations occurring despite gut hormone suppression. This is important to bear in mind when interpreting these findings and could feasibly underlie the lack of a positive effect with regards to body weight and composition with treatment. This is particularly pertinent as the studies describing an impact of short-acting Octreotide did demonstrate significant GLP-1 attenuation $(27,28)$. There was no clear precedent on which to base the dosing in this clinical context. The 10mg dose was selected based on weighing the risks and potential benefits of treatment. In an already nutritionally vulnerable cohort, the ethical principle of non-maleficence guided us in choosing a dose less likely to elicit adverse effects. In a study of patients post gastrectomy, 10mg of Octreotide was sufficient to improve quality of life within four weeks and those requiring up-titration to $20 \mathrm{mg}$ were less likely to derive benefit overall (30). As such, in the clinical context, patients were commenced on a $10 \mathrm{mg}$ dose, and this trial of therapy continued for one month at which point a shared decision was made with the patient based on subjective and objective treatment benefit. Future longerterm studies, allowing dose titration to avoid side effects, will be required to determine whether a prolonged duration of treatment and/or higher doses of Octreotide may induce observable changes in eating behaviour and body composition.

Post-prandial hypoglycemia is a phenomenon that occurs after foregut surgery and may be related to exaggerated post-prandial gut hormone release $(43,44)$. In the current study, however, there were no cases of biochemical or symptomatic hypoglycemia post-prandially. Nonetheless, a minimally potent dose of a long-acting somatostatin analogue was associated with an increase in post-prandial plasma glucose concentrations and a corresponding alteration of the pattern of post-prandial plasma insulin dynamics, delaying its peak.

GLP-1 exerts its anorectic effects in the hypothalamic arcuate nucleus, but may also play a role in the 
hedonic signaling pathways of food reward $(12,13,17,45)$. Indeed, in related surgeries, such as Roux-en-Y gastric bypass (RYGB) surgery, attenuation of the exaggerated post-prandial GLP-1 (and PYY) gut hormone secretion is associated with increased brain reward system food cue reactivity and food appeal (12). This study used an established fMRI paradigm to assess food cue reactivity $(12,36-$ 38), assessing BOLD signal in a priori regions of interest within the brain reward system, to explore the relationship of medium-term Octreotide LAR therapy with anticipatory food reward, but did not find a significant increase, or even a trend for an increase, in BOLD signal to high-energy or lowenergy foods to corroborate previous findings. However, the degree of plasma gut hormone suppression achieved was notably less, and as such these findings are not presented as an attempt to rebut previous work on this question. Future studies will need to explore the use of higher doses to ascertain the potential role for long-acting Octreotide in improving outcomes by influencing food reward signaling, as well as increasing the number of participants.

Eating behavior is the final step along the pathway assessed in this study. This comprised the PRT, exploring appetitive behavior, and an ad libitum buffet meal, to directly measure food intake and food selection. The latter is subject to external conditioned and/or unconditioned influences, such as postprandial symptoms creating food avoidance, or social cues or contexts, respectively $(21,46)$. As noted, previous work in patients after esophagectomy reported a 1.5-fold increase in ad libitum energy intake and increased motivation to receive a sweet-fat stimulus in the fed state with acute Octreotide administration $(27,28)$, mirroring similar observations in RYGB cohorts (12). The findings outlined in this paper suggest there is an association between the degree of gut hormone suppression obtained and alterations in eating behavior, as those with greater plasma GLP-1 suppression were more likely to increase their motivation to receive a food reward. However, this finding did not translate into a subsequent increase in energy intake in these participants during the buffet meal.

The ad libitum buffet meal did not corroborate previous findings. However, along with the suboptimal 
plasma GLP-1 suppression, the methodology previously utilized differed. This study utilized a realworld buffet style meal based on patient preferences, while previous work has used a method involving investigator-controlled and timed provision of a single food item until satiation was reached by the participant. This distinction introduces variables such as conditioned food avoidance, a phenomenon whereby learned behavior over time in an attempt to mitigate against post-ingestive symptoms can lead to active avoidance of certain foods or food quantities (46). Interestingly, and relevant to the complexity and myriad factors involved in eating behavior, at the baseline visit, energy intake was not significantly associated with the relative or absolute increase in plasma GLP-1 at the meal suggesting that participant's food ingestion was more strongly influenced by other factors besides satiety signaling.

Prior longitudinal work in an esophagectomy cohort has shown that the exaggerated enteroendocrine response corresponds with important appetitive symptoms, such as early satiety, and correlates with Sigstad score (11). In this study, treatment with Octreotide LAR was associated with a $30 \%$ relative reduction in Sigstad score, reflective of acute post-prandial symptom burden. Although limited by the subjective nature of questionnaire data, if this potential finding is validated in larger studies, this represents a promising option for the management of postprandial symptoms. Indeed, prolonged exposure to a reduced symptom profile may manifest in more profound positive changes in eating behavior and body weight over time.

Several limitations must be acknowledged, which should be viewed in the context of this as a pilot study. This was not a randomized or controlled study, thus findings - positive and negative - must be interpreted with caution, recognizing its inability to infer causality. Given the relatively small sample size, negative results must be interpreted with caution as a Type II error cannot be excluded. The unblinded uncontrolled design exposes these findings to the order effect, whereby participants may improve performance on repeat testing based on prior experience. Notwithstanding this, the gross 
absence of differences in VAS scores between visits does indicate that the potential of bias at the buffet meal may be less likely. As noted, the degree of gut hormone suppression achieved with clinical treatment was less than expected, which also limits the interpretation of these findings. This factor precludes the representation of any observations herein as associated with significant attenuation of the post-prandial gut hormone response. We have discussed them instead in the context of Octreotide LAR therapy. However, this absence of evidence of suppression at the four-week timepoint does not serve as evidence of absence of suppression at some point earlier in the treatment period.

In conclusion, this pilot study outlines, in a post-esophagectomy cohort with significant weight loss, the parallel characterization of multiple levels of the gut-brain axis and eating behavior, which will benefit future work aiming to better elucidate the role of these processes in the etiology of postoperative malnutrition. Moreover, it provided insight into the impact of a 4 week 10mg Octreotide LAR treatment course on the postprandial gut hormone response, food reward, and eating behavior. Though gut hormone suppression was suboptimal, patients did not exhibit altered body weight or appetitive behavior. These findings may be used to inform the design of future studies investigating the value of gut hormone attenuation as a therapy for malnutrition and weight loss, while also improving our mechanistic understanding to facilitate the development of more targeted therapeutic strategies. 


\section{Acknowledgements}

The authors would like to acknowledge the study participants, as well as the support and contributions of the Centre for Advanced Medical Imaging, St James's Hospital, Dublin, Ireland; and the HRBWellcome Trust-Clinical Research Facility, St. James’s Hospital, Dublin, Ireland.

\section{Data Availability}

The datasets generated during and/or analyzed during the current study are not publicly available but are available from the corresponding author on reasonable request. 


\section{REFERENCES}

1. Martin L, Lagergren J, Lindblad M, et al. Malnutrition after oesophageal cancer surgery in Sweden. Br J Surg. 2007;94(12):1496-500.

2. Martin L, Lagergren P. Long-term weight change after oesophageal cancer surgery. Br J Surg. 2009;96(11):1308-14.

3. Markar SR, Zaninotto G, Castoro C, et al. Lasting Symptoms After Esophageal Resection (LASER): European Multicenter Cross-sectional Study. Annals of Surgery. 2020; Published online ahead of print.

4. Boshier PR, Klevebro F, Savva KV, et al. Assessment of Health Related Quality of Life and Digestive Symptoms in Long-term, Disease Free Survivors After Esophagectomy. Annals of Surgery. 2020; Published online ahead of print.

5. Elliott JA, Doyle SL, Murphy CF, et al. Sarcopenia: prevalence, and impact on operative and oncologic outcomes in the multimodal management of locally advanced esophageal cancer. Ann Surg. 2017;266(5):822-30.

6. Elliott JA, Casey S, Murphy CF, et al. Risk factors for loss of bone mineral density after curative esophagectomy. Archives of Osteoporosis. 2019;14:6.

7. Heneghan HM, Zaborowski A, Fanning M, et al. Prospective Study of Malabsorption and Malnutrition After Esophageal and Gastric Cancer Surgery. Ann Surg. 2015;262(5):803-7; discussion $7-8$.

8. Guinan E, Bennett A, Doyle S, et al. Measuring the impact of oesophagectomy on physical functioning and physical activity participation: a prospective study. BMC cancer. 2019;19(1):682.

9. Hellstadius Y, Malmström M, Lagergren P, et al. Reflecting a crisis reaction: Narratives from patients with oesophageal cancer about the first 6 months after diagnosis and surgery. Nursing Open. 2019;6(4):1471-80.

10. Anandavadivelan P, Wikman A, Johar A, et al. Impact of weight loss and eating difficulties on health- related quality of life up to 10 years after oesophagectomy for cancer. Br J Surg. 2018;105(4):410-8. 
11. Elliott J, Docherty N, Murphy C, et al. Changes in gut hormones, glycaemic response and symptoms after oesophagectomy. Br J Surg. 2019;106(6):735-46.

12. Goldstone AP, Miras AD, Scholtz S, et al. Link Between Increased Satiety Gut Hormones and Reduced Food Reward After Gastric Bypass Surgery for Obesity. J Clin Endocrinol Metab. 2016;101(2):599-609.

13. Miras AD, le Roux CW. Mechanisms underlying weight loss after bariatric surgery. Nat Rev Gastroenterol Hepatol. 2013;10(10):575.

14. le Roux CW, Bueter M. The physiology of altered eating behaviour after Roux-en-Y gastric bypass. Exp Physiol. 2014;99(9):1128-32.

15. Lenard NR, Berthoud H-R. Central and Peripheral Regulation of Food Intake and Physical Activity: Pathways and Genes. Obesity (Silver Spring, Md). 2008;16(Suppl 3):S11-S22.

16. Ochner CN, Kwok Y, Conceição E, et al. Selective Reduction in Neural Responses to High Calorie Foods Following Gastric Bypass Surgery. Annals of surgery. 2011;253(3):502-7.

17. Behary P, Miras AD. Food preferences and underlying mechanisms after bariatric surgery. Proc Nutr Soc. 2015;74(4):419-25.

18. Rolls ET. Taste, olfactory and food texture reward processing in the brain and the control of appetite. The Proceedings of the Nutrition Society. 2012;71(4):488-501.

19. Grill HJ, Kaplan JM. The neuroanatomical axis for control of energy balance. Frontiers in neuroendocrinology. 2002;23(1):2-40.

20. Robinson TE, Berridge KC. The neural basis of drug craving: an incentive-sensitization theory of addiction. Brain Res Brain Res Rev. 1993;18(3):247-91.

21. Castro DC, Berridge KC. Advances in the neurobiological bases for food 'liking' versus 'wanting'. Physiol Behav. 2014;136:22-30.

22. Hill RJ, Davies PS. The validity of self-reported energy intake as determined using the doubly labelled water technique. Br J Nutr. 2001;85(4):415-30.

23. Schoeller DA. Limitations in the assessment of dietary energy intake by self-report. Metabolism. 1995;44(2 Suppl 2):18-22. 
24. Trabulsi J, Schoeller DA. Evaluation of dietary assessment instruments against doubly labeled water, a biomarker of habitual energy intake. Am J Physiol Endocrinol Metab. 2001;281(5):E891-9.

25. Mathes CM, Spector AC. Food selection and taste changes in humans after Roux-en-Y gastric bypass surgery: a direct-measures approach. Physiol Behav. 2012;107(4):476-83.

26. Gero D, Steinert RE, le Roux CW, et al. Do Food Preferences Change After Bariatric Surgery? Current atherosclerosis reports. 2017;19(9):38.

27. Elliott JA, Jackson S, King S, et al. Gut Hormone Suppression Increases Food Intake After Esophagectomy With Gastric Conduit Reconstruction. Ann Surg. 2015;262(5):824-30.

28. Elliott JA, Docherty NG, Haag J, et al. Attenuation of satiety gut hormones increases appetitive behavior after curative esophagectomy for esophageal cancer. Am J Clin Nutr. 2019;109(2):335-44.

29. Geer RJ. Efficacy of octreotide acetate in treatment of severe postgastrectomy dumping syndrome. Ann Surg. 1990;212:678-87.

30. Penning C, Vecht J, Masclee AA. Efficacy of depot long-acting release octreotide therapy in severe dumping syndrome. Aliment Pharmacol Ther. 2005;22(10):963-9.

31. Woodend DM, Anderson GH. Effect of sucrose and safflower oil preloads on short term appetite and food intake of young men. Appetite. 2001;37(3):185-95.

32. Sigstad H. A clinical diagnostic index in the diagnosis of the dumping syndrome. Changes in plasma volume and blood sugar after a test meal. Acta Med Scand. 1970;188:479-86.

33. Aaronson NK, Ahmedzai S, Bergman B, et al. The European Organization for Research and Treatment of Cancer QLQ-C30: a quality-of-life instrument for use in international clinical trials in oncology. J Natl Cancer Inst. 1993;85(5):365-76.

34. Ryan AM, Reynolds JV, Healy L, et al. Enteral nutrition enriched with eicosapentaenoic acid (EPA) preserves lean body mass following esophageal cancer surgery: results of a double-blinded randomized controlled trial. Ann Surg. 2009;249(3):355-63.

35. Ryan AM, Rowley SP, Healy LA, et al. Post-oesophagectomy early enteral nutrition via a needle catheter jejunostomy: 8-year experience at a specialist unit. Clin Nutr. 2006;25(3):386-93. 
36. Byrne CS, Chambers ES, Alhabeeb $\mathrm{H}$, et al. Increased colonic propionate reduces anticipatory reward responses in the human striatum to high-energy foods. Am J Clin Nutr. 2016;104(1):5-14.

37. Scholtz S, Miras AD, Chhina N, et al. Obese patients after gastric bypass surgery have lower brain-hedonic responses to food than after gastric banding. Gut. 2014;63(6):891-902.

38. Goldstone AP, Prechtl CG, Scholtz S, et al. Ghrelin mimics fasting to enhance human hedonic, orbitofrontal cortex, and hippocampal responses to food. Am J Clin Nutr. 2014;99(6):131930.

39. Murphy CF, Stratford N, Docherty NG, et al. AttenuatingGutBrainSignalling_Supplemental Material. Figshare. Journal contribution. https://figshare.com/s/f8a9b1373c19fb5071bd. 2020.

40. Geiselman PJ, Anderson AM, Dowdy ML, et al. Reliability and validity of a macronutrient self-selection paradigm and a food preference questionnaire. Physiol Behav. 1998;63(5):919-28.

41. Ritz P, Hanaire H. Post-bypass hypoglycaemia: a review of current findings. Diabetes Metab. 2011;37(4):274-81.

42. Geer RJ, Richards WO, O'Dorisio TM, et al. Efficacy of octreotide acetate in treatment of severe postgastrectomy dumping syndrome. Ann Surg. 1990;212(6):678-87.

43. Tack J, Arts J, Caenepeel P, et al. Pathophysiology, diagnosis and management of postoperative dumping syndrome. Nat Rev Gastroenterol Hepatol. 2009;6(10):583.

44. Gebhard B, Holst JJ, Biegelmayer C, et al. Postprandial GLP-1, norepinephrine, and reactive hypoglycemia in dumping syndrome. Dig Dis Sci. 2001;46(9):1915-23.

45. Murphy KG, Bloom SR. Gut hormones in the control of appetite. Exp Physiol. 2004;89(5):507-16.

46. Schier LA, Spector AC. The functional and neurobiological properties of bad taste. Physiological reviews. 2018;99(1):605-63. 


\section{FIGURE LEGENDS}

Figure 1. Study protocol flowchart for 'REWARD' study.

fMRI, functional magnetic resonance imaging; HR-QL, health-related quality of life; mGSRS, modified gastrointestinal rating scale; LAR, long-acting release. PRT, progressive ratio task.

Figure 2. Post-prandial plasma GLP-1 before and after treatment with octreotide LAR.

(A) Post-prandial plasma total GLP-1 secretion, (B) peak postprandial GLP-1, and (C) total postprandial area under curve (AUC) from before to 150 minutes, and (D) early AUC of post-prandial GLP-1 secretion before and after treatment with Octreotide LAR $(n=8)$. Panel A: The grey dotted line represents consumption of the standardized $400 \mathrm{kcal}$ mixed meal. Repeated measures two-way ANOVA with post-hoc Bonferroni's multiple comparisons test: mean difference [95\% confidence interval] $8.47[-0.91,17.85], \mathrm{F}[1,42]=3.32, P=0.076$, Cohen's $\mathrm{d}=-0.23 . \mathrm{T}=30$, mean difference [95\% CI] 53.37 [21.85, 84.89, $P<0.001]$. Panel B: Mean \pm SEM, paired $t$ test: mean difference [95\% $\mathrm{CI}]-21.81[-45.33,1.70], \mathrm{t}=2.19, \mathrm{df}=7, P=0.064$, Cohen's $\mathrm{d}=-0.44$. Panel C: Mean \pm SEM, paired $t$ test: mean difference [95\% CI] $-44.61[-90.09,0.87], \mathrm{t}=2.32, \mathrm{df}=7, P=0.053$, Cohen's $\mathrm{d}=-0.75$. Panel D: Mean \pm SEM, paired $t$ test: mean difference [95\% CI] $-43.93[-137.3,49.4], \mathrm{t}=1.11, \mathrm{df}=7$, $P=0.30$, Cohen's $\mathrm{d}=-0.42$. Abbreviations: GLP-1, glucagon-like peptide-1.

\section{Figure 3. Post-prandial plasma glucose and insulin before and after treatment with octreotide}

\section{LAR.}

Time course of plasma (A) glucose and (B) insulin before and after standardized $400 \mathrm{kcal}$ mixed meal with grey dotted line representing time of meal consumption (n=8). (A) Post-prandial plasma glucose concentrations were higher after treatment with octreotide LAR. 2-way repeated measures ANOVA with Bonferroni’s multiple comparisons test: mean difference [95\% confidence interval] -0.385 [- 
$0.763,-0.007], \mathrm{F}[1,42]=4.23, P=0.046$, Cohen's $\mathrm{d}=0.26$. (B) There was a treatment $\mathrm{x}$ time effect on post-prandial plasma insulin after treatment with a delayed peak, but no overall difference in postprandial concentrations after treatment: mean difference [95\% CI] $-1.454[-8.791,5.883], \mathrm{F}[1,42]=$ $0.16, P=0.69$, Cohen's $d=0.04$. At $\mathrm{T}=30$, post-treatment insulin concentration was significantly lower (mean difference [95\% CI]: 53.29 [28.63, 77.95], $P<0.001$ ), while at T=60 insulin was significantly higher after treatment (mean difference [95\% CI]: -57.89 [-82.55 to -33.23$], P<0.001$ ).

Figure 4. Progressive ratio task before and after treatment with octreotide LAR.

(A) Breakpoint and (B) total clicks in progressive ratio task (PRT) before and after treatment with octreotide LAR. Data given as median \pm IQR (interquartile range), $n=8$. (A) Wilcoxon matched pairs signed rank test: $P=0.41$, Cohen's $d=-0.47$, (B) mean \pm SEM, paired t test: mean difference $[95 \%$ $\mathrm{CI}]-228[-1030,575.2], \mathrm{t}=0.67, \mathrm{df}=7, P=0.52$, Cohen's $\mathrm{d}=-0.32$.

Figure 5. Ad libitum buffet meal before and after treatment with octreotide LAR.

Octreotide LAR treatment was not associated with (A) a change in total energy intake at ad libitum buffet meal (mean \pm SEM, paired t test: mean difference [95\% CI] $50.65[-101.0,202.3], \mathrm{t}=0.79$, df $=7, P=0.46$ ), nor (B) macronutrient selection at ad libitum buffet meal 2-way (repeated measures ANOVA with post-hoc Bonferroni's multiple comparisons test: mean difference [95\% confidence interval] $-0.095[-1.604,1.414], \mathrm{F}[1,28]=0.02, P=0.89$, Cohen's $\mathrm{d}<0.01) ; \mathrm{n}=8$. Abbreviations: $\mathrm{CHO}$, complex carbohydrates.

Figure 6. Food picture evaluation and auditory-motor-visual fMRI tasks before and after treatment with octreotide LAR. 
There were no significant differences in the magnitude of BOLD signal (\%) in a priori regions of interest ROI) in the (A-D) food picture evaluation functional MRI task (amygdala, anterior insula, orbitofrontal cortex (OFC), putamen, nucleus accumbens, and caudate) before vs. after treatment for contrasts: (A) high-energy vs. low-energy food, mean difference [95\% confidence interval] 0.038 [$0.096-0.173], F[1,7]=0.46, P=0.52$, Cohen's $d=-0.55$, (B) high-energy or low-energy foods (vs. objects), mean difference [95\% CI] $0.079[-0.032,0.191], \mathrm{F}[1,7]=2.82, P=0.14$, Cohen's $\mathrm{d}=-1.65$, (C) high-energy foods (vs. objects), mean difference [95\% CI $0.097[-0.007,0.200], \mathrm{F}[1,7]=4.88$, $P=0.06$, Cohen's $\mathrm{d}=-1.54$, and (D) low-energy foods (vs. objects), mean difference $[95 \% \mathrm{CI}] 0.062$ $[-0.094,0.218], \mathrm{F}[1,7]=0.89, P=0.38$, Cohen's $\mathrm{d}=-1.14$, nor $(\mathrm{E})$ auditory-motor-visual control functional MRI task (bilateral superior temporal gyrus in auditory task, left precentral gyrus in motor task, and bilateral lingual gyrus in visual task), nor (F) appeal rating of low-energy or high-energy foods (vs. objects) in food picture evaluation functional MRI task. Analyses used 2-way repeated measures ANOVA including (A-E) visit and ROI, or (F) visit and energy density, as within subject factors with post-hoc Fisher least-significant difference test $(n=8)$. Abbreviations: Amy, amygdala; AMV, auditory-motor-visual; BOLD, blood oxygen level-dependent; Caud, caudate; ED, energy density; HE, high-energy; Ins, anterior insula; LE, low-energy; Lingual, lingual gyrus; LpreCG, left precentral gyrus; NAcc, nucleus accumbens; OFC, anterior orbitofrontal cortex; Put, putamen; STG, superior temporal gyrus posterior division.

Figure 7. Sigstad dumping syndrome score before and after treatment with octreotide LAR.

Sigstad score was significantly lower after 4 weeks of treatment. Median \pm interquartile range, Wilcoxon matched-pair signed rank test: $P=0.039$, Cohen's $\mathrm{d}=-0.61, \mathrm{n}=8$. 
Table 1: Clinicopathological characteristics of the study cohort

Cohort

$N=8$

\section{Clinical Characteristics}

Age, mean (SD)

$62.8(9.4)$

Sex, n (\%)

Female

Male

Body weight at $1^{\text {st }}$ study visit, $\mathrm{kg}$, mean (SD)

BMl at $1^{\text {st }}$ study visit, $\mathrm{kg} / \mathrm{m}^{2}$, mean (SD)

Pre-illness BMI, $\mathrm{kg} / \mathrm{m}^{2}$, mean (SD)

Preoperative BMI, $\mathrm{kg} / \mathrm{m}^{2}$, mean (SD)

Post-diagnosis body weight loss (\%), mean (SD)

Post-operative body weight loss (\%), mean (SD)

Time since operation, months, median (range)

\section{Pathology}

Adenocarcinoma, n (\%)

$6(75.0)$

SCC, n (\%)

$2(25.0)$

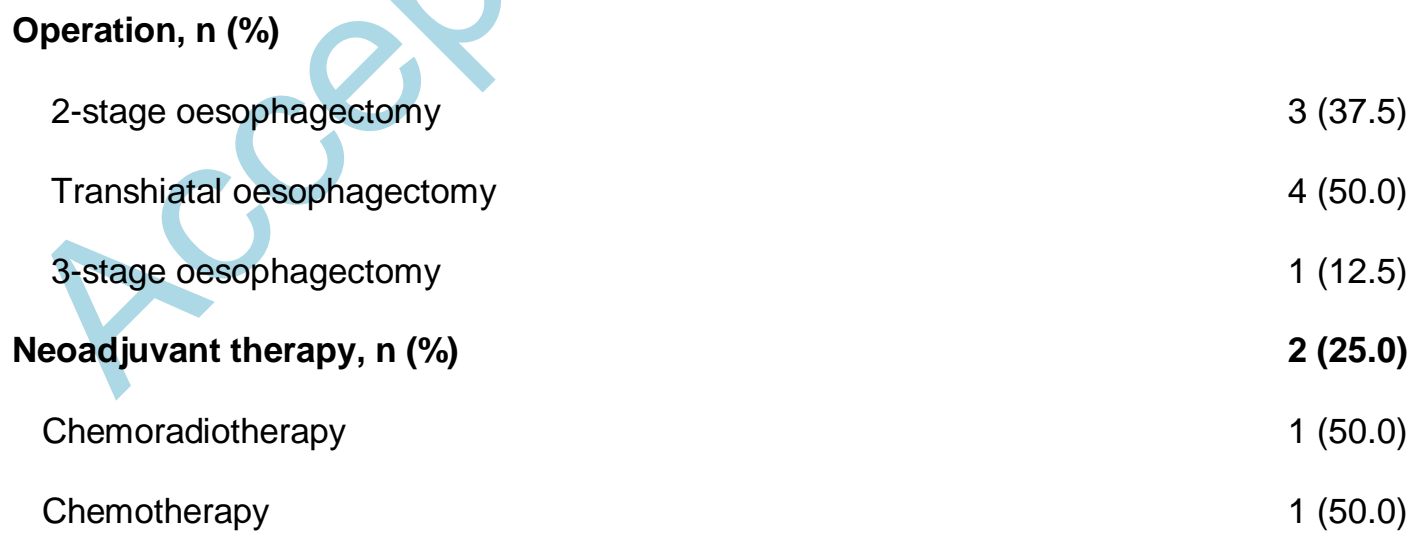

Transhiatal oesophagectomy

3-stage oesophagectomy

Neoadjuvant therapy, n (\%)

Chemoradiotherapy

$1(50.0)$

Chemotherapy

1 (50.0)

Abbreviations: BMI, body mass index $\left(\mathrm{kg} \mathrm{m}^{-2}\right)$; SCC, squamous cell carcinoma; $S D$, standard deviation. 
Table 2. Body weight, composition, and anthropometry in patients before and after treatment with long-acting Octreotide.

Week $0 \quad$ Week 4

$$
\mathrm{N}=8 \quad \mathrm{~N}=8 \quad P \text {-value }
$$

\section{Anthropometry}

Body weight, $\mathrm{kg}$, mean (SD)

$68.6(12.8)$

$69.2(13.4)$

0.129

BMI, $\mathrm{kg} / \mathrm{m}^{2}$, mean (SD)

$22.4(2.6)$

$22.7(3.0)$

0.207

$\%$ Body weight change, mean (SD)

$0.68(1.3) \bigcirc 0.185^{\star}$

Mid-upper arm circumference, $\mathrm{cm}$, mean (SD)

$25.6(1.4)$

$25.8(2.4)$

0.785

Waist:Hip Ratio, mean (SD)

$0.88(0.1)$

$0.90(0.1)$

0.024

\section{Body Composition}

Skeletal muscle mass, kg, mean (SD)

$25.4(5.8)$

$25.5(6.1)$

0.914

Fat-free mass, kg, mean (SD)

$52.7(9.8)$

$52.6(10.2)$

0.965

Fat mass, kg, mean (SD)

$16.0(4.9)$

$16.6(5.0)$

0.406

Fat mass, \%, mean (SD)

$23.3(5.2)$

$23.8(5.3)$

0.582

Paired t test unless otherwise specified. *one-sample t test. Abbreviations: BMI, body mass index; SD, standard deviation. 
Table 3. Selected health-related quality of life and nutrition-specific symptoms before and after treatment with Octreotide LAR

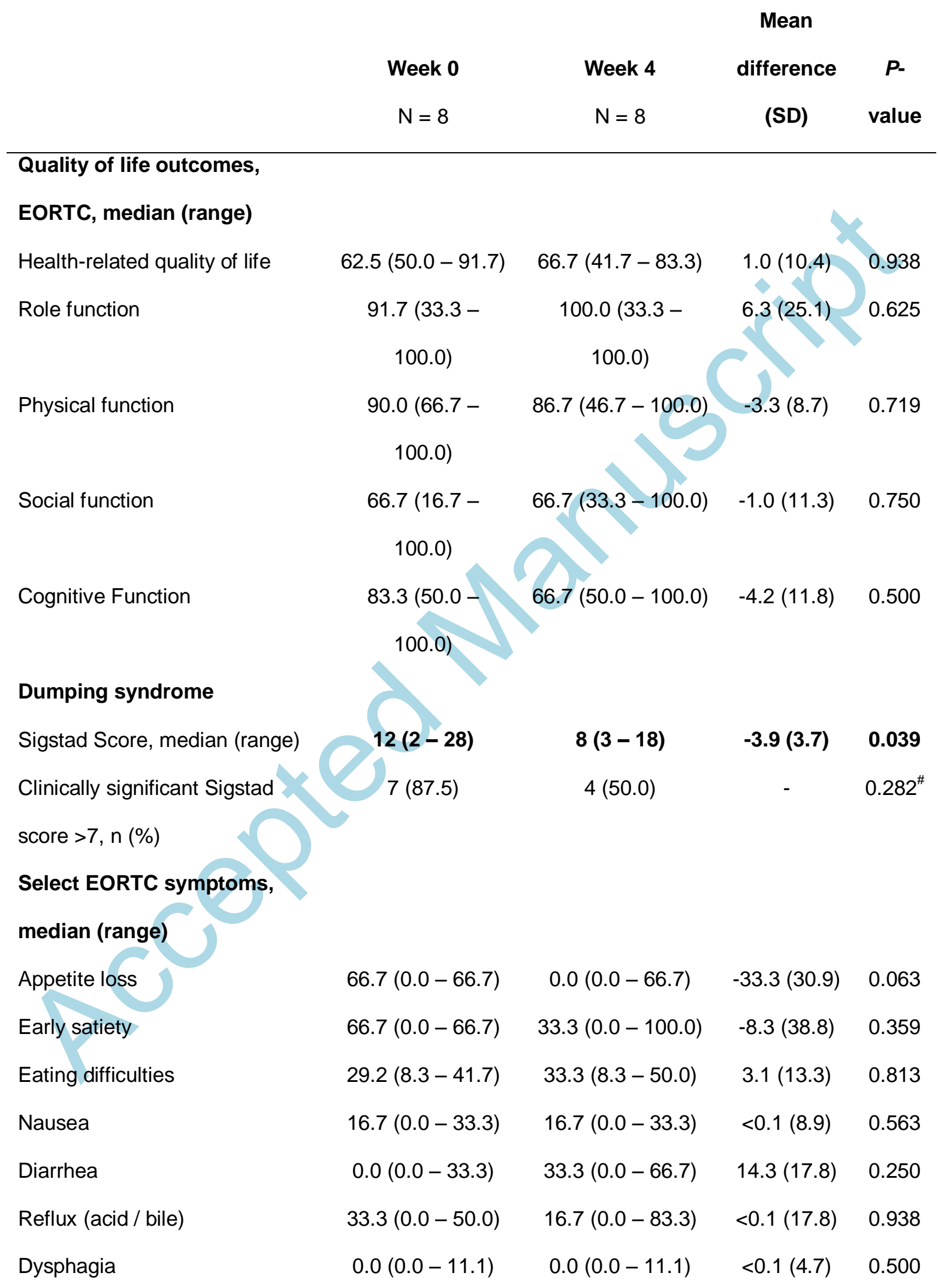

All analyses used Wilcoxon matched-pairs signed rank tests, unless otherwise specified: ${ }^{\#}$ Fisher's 
exact test. For quality of life outcomes, possible scores range from $0-100$, with 100 representing an optimal score. Abbreviations: EORTC, European Organization for Research and Treatment of Cancer; $S D$, standard deviation. 
Figure 1

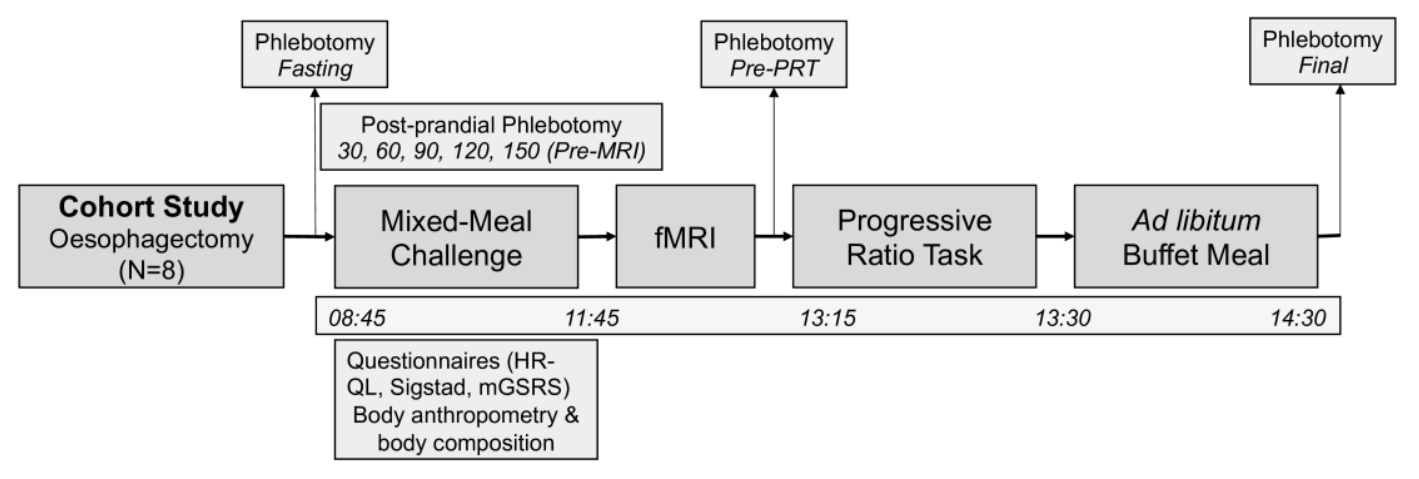


Figure 2

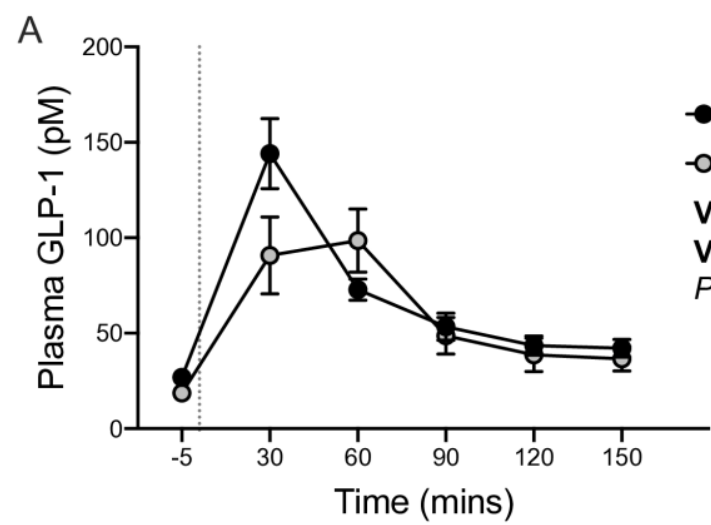

C

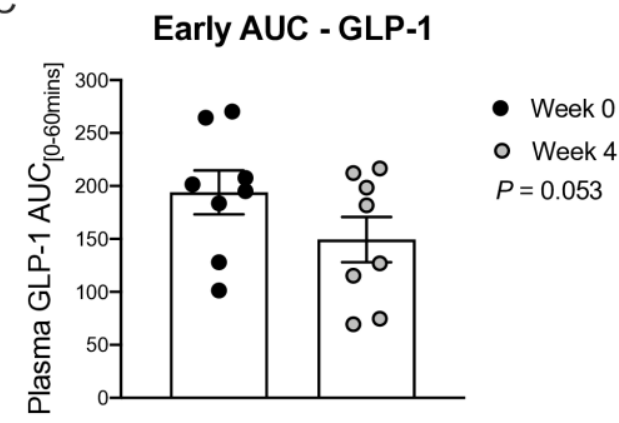

B

- Week 0

Visit: $P=0.076$ Visit * Time:

$P=0.011$

D

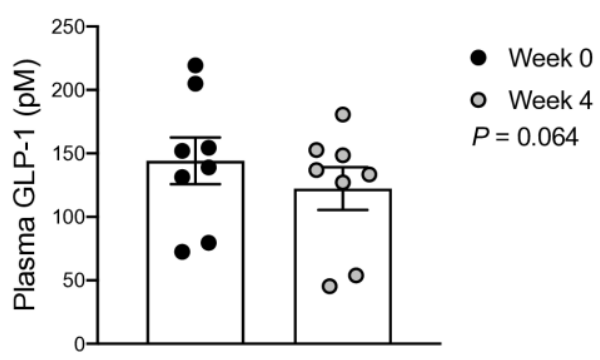

Total AUC - GLP-1

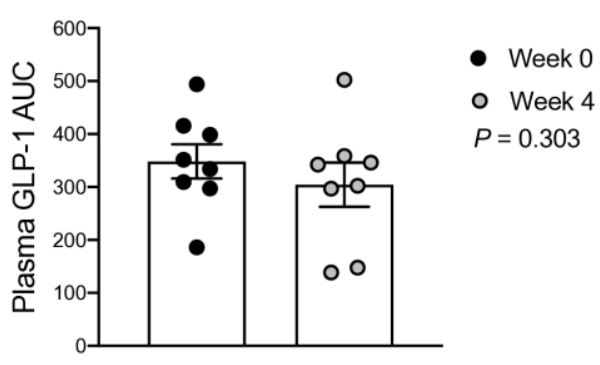


Figure 3

A

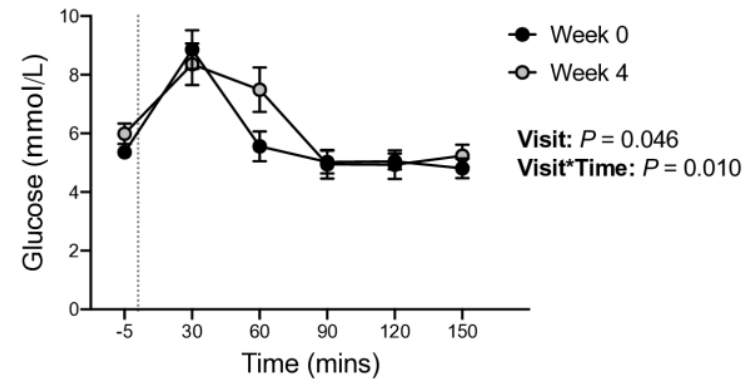

B

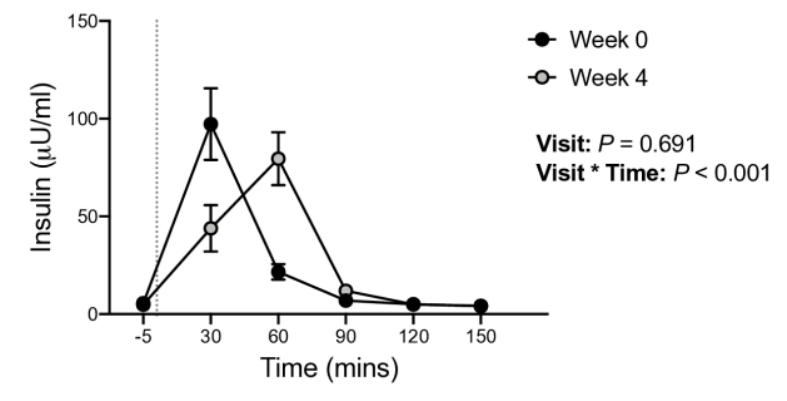


Figure 4
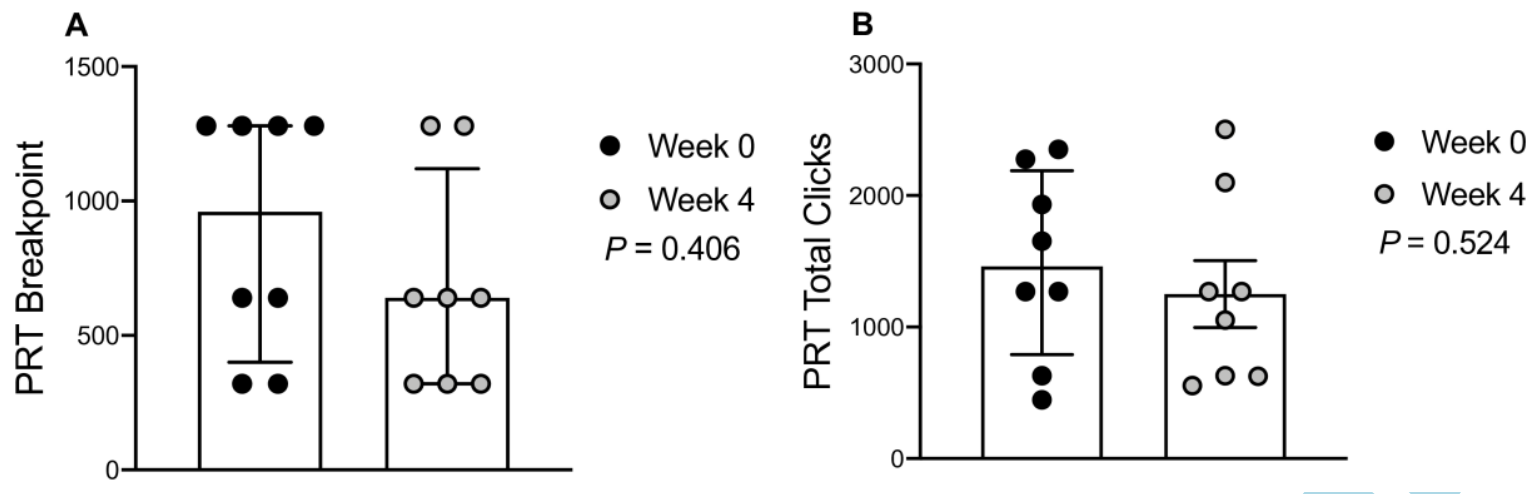
Figure 5

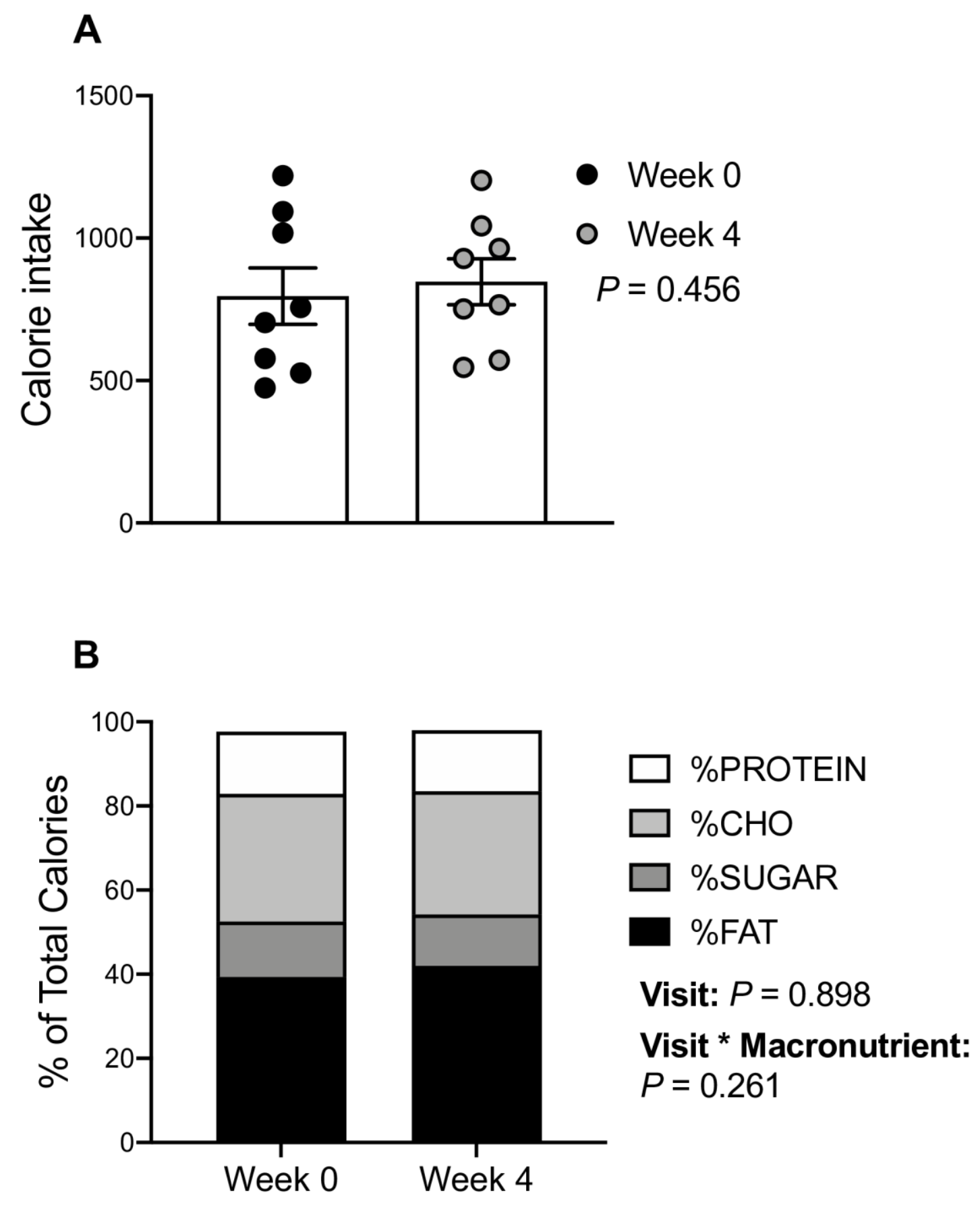


A

High vs. low-energy food

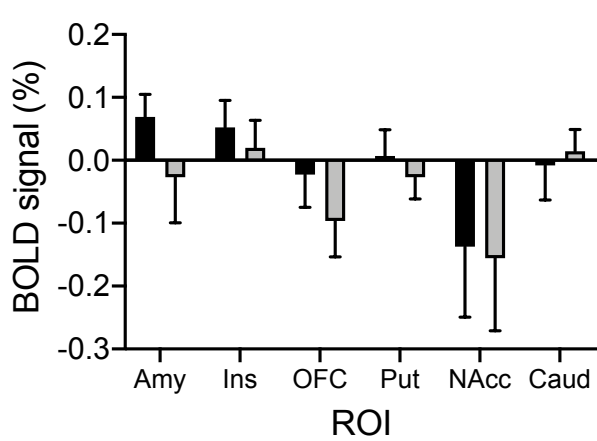

C

High-energy food vs. Object

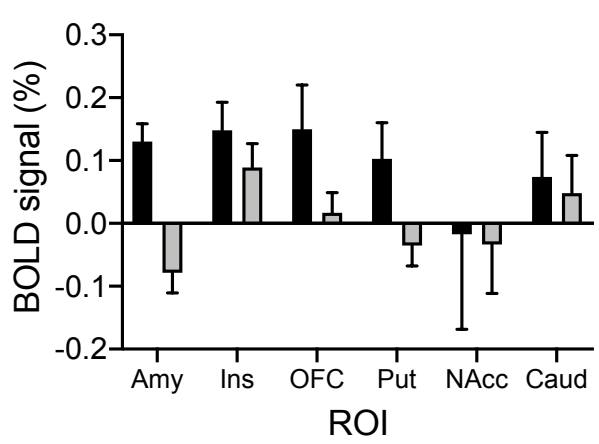

E

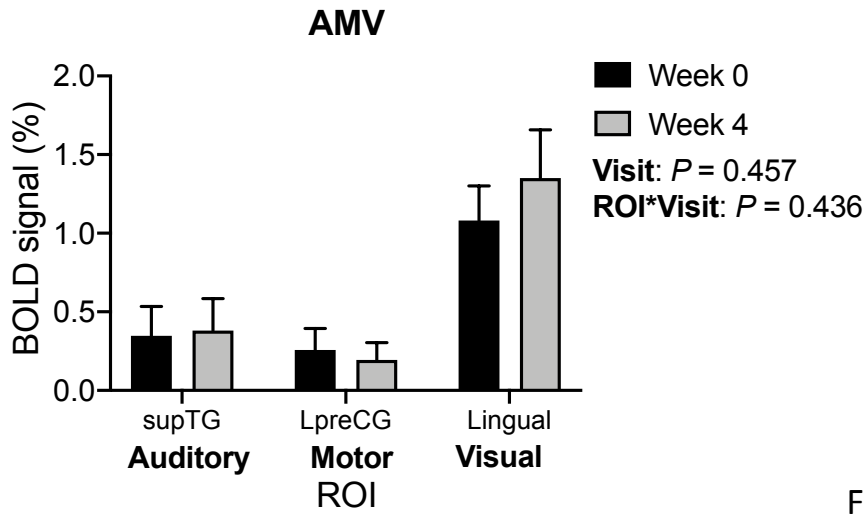

B

Food vs. Object

Week 0

$\square$ Week 4

Visit: $P=0.519$

ROI*Visit: $P=0.525$

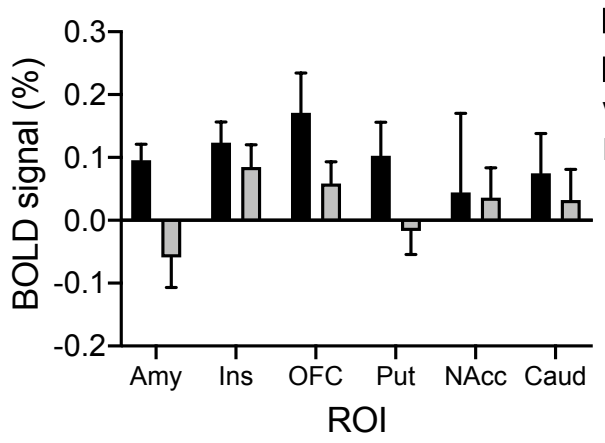

D

Week 0

Week 4

Visit: $P=0.063$

ROI*Visit: $P=0.141$
Low-energy food vs. Object

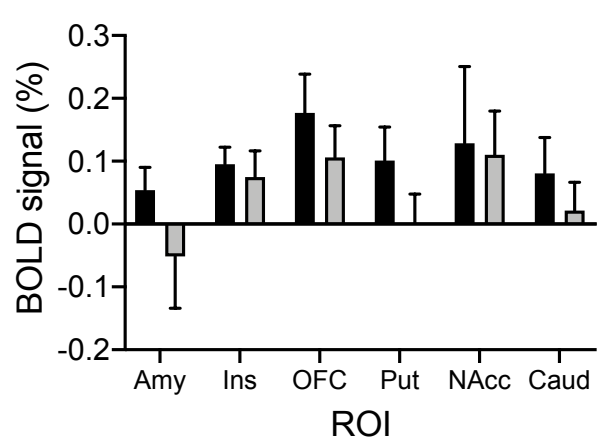

Week 0

Week 4

Visit: $P=0.376$

ROI*Visit: $P=0 . \bar{\not} 34$

F

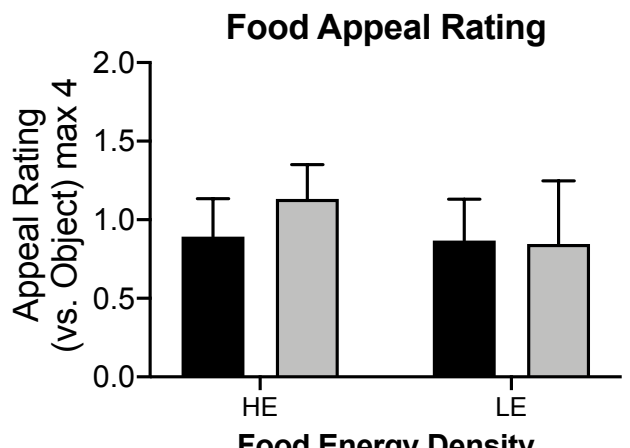

Week 0

Week 4

Visit: $P=0.496$

ED*Visit: $P=0.06$

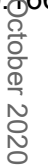




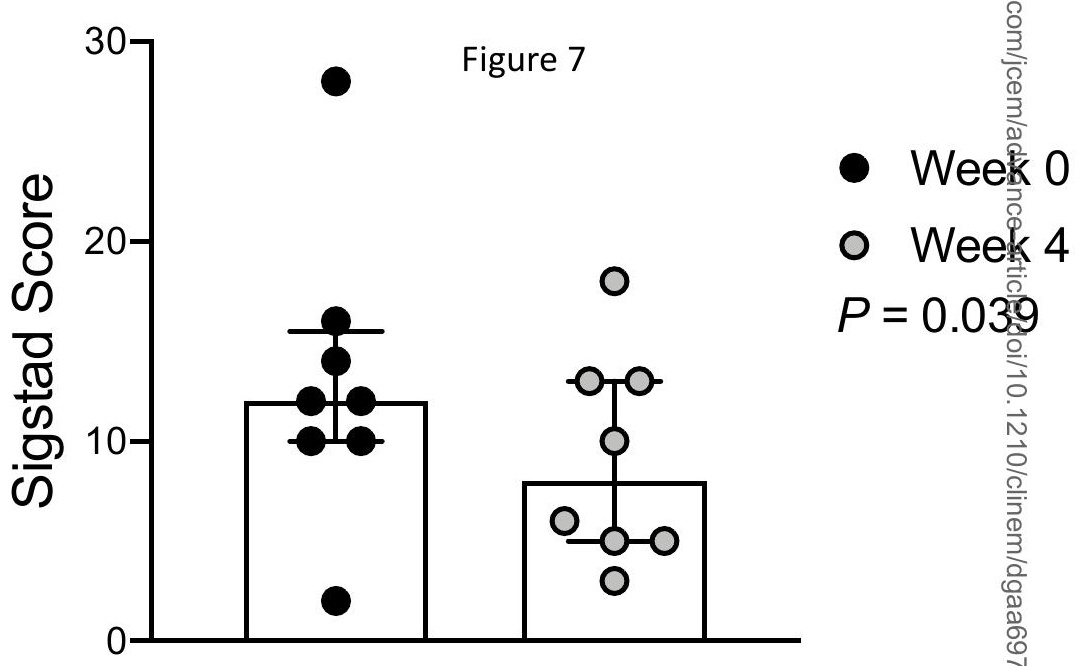

